\title{
Differences in the antimicrobial susceptibility profiles of Moraxella bovis, M. bovoculi and M. ovis
}

\author{
Grazieli Maboni ${ }^{1}$, Leticia T. Gressler ${ }^{1}$, Julia P. Espindola ${ }^{1}$, Marcelo Schwab ${ }^{1}$, \\ Caiane Tasca ${ }^{1}$, Luciana Potter ${ }^{2}$, Agueda Castagna de Vargas ${ }^{1}$ \\ ${ }^{1}$ Departamento de Medicina Veterinária Preventiva, Universidade Federal de Santa Maria, \\ Santa Maria, RS, Brazil. \\ ${ }^{2}$ Departamento de Zootecnia, Universidade Federal de Santa Maria, Santa Maria, RS, Brazil.
}

\begin{abstract}
The aim of this study was to determine the differences in the antimicrobial susceptibility profiles of Moraxella bovis, M. bovoculi and M. ovis. Thirty-two strains of Moraxella spp. isolated from cattle and sheep with infectious keratoconjunctivitis were tested via broth microdilution method to determine their susceptibility to ampicillin, cefoperazone, ceftiofur, cloxacillin, enrofloxacin, florfenicol, gentamicin, neomycin, oxytetracycline and penicillin. The results demonstrated that Moraxella spp. strains could be considered sensitive for most of the antimicrobials tested in this study, but differences between the antimicrobial susceptibility profiles of these three Moraxella species were found. M. bovis might differ from other species due to the higher MIC and MBC values it presented.
\end{abstract}

Key words: bacterial resistance, broth microdilution, eye disease.

\section{Introduction}

Infectious keratoconjunctivitis (IK) affects cattle and sheep and is characterized by the development of conjunctivitis and corneal ulcers (Baptista, 1979). In cattle, the microorganisms involved in IK are M. bovis (Henson and Grumbles, 1960), M. ovis (Elad et al., 1988) and M. bovoculi (Angelos et al., 2007). In contrast, in sheep, the main microorganisms isolated from IK lesions are Mycoplasma conjunctivae, Chlamydophila psittaci and $M$. ovis (Elad et al., 1988; Dagnall, 1994). M. bovis can also be isolated from sheep but it is found at a lower frequency (Libardoni et al., 2012). The treatment of this disease is based on antimicrobial therapy, which should be adopted considering that it is necessary to combat two or more species of Moraxella present in the same lesion. However, studies addressing the antimicrobial susceptibility of $M$. bovis, M. bovoculi and M. ovis are scarce in the literature. To the best of our knowledge, this is the first study to report the antimicrobial susceptibility of M. ovis isolated from IK in sheep and the first study in Brazil to describe the antimicrobial susceptibility of $M$. bovoculi.
It is important to determine the antimicrobial susceptibility of Moraxella spp., so that if the disease occurs the best-possible treatment will be provided. In this context, the aim of this study was to determine the differences in the antimicrobial susceptibility profiles of $M$. bovis, $M$. bovoculi and M. ovis.

\section{Materials and Methods}

\section{Samples characterization}

Samples from clinical cases of infectious bovine and ovine IK occurring in southern Brazil were previously processed. These samples included different strains from the same herd, from different herds in similar locations and from different locations. A total of 32 samples were characterized as Moraxella spp. based on Gram staining and biochemical tests (Macfaddin, 2000). To perform molecular identification of species, DNA was extracted using the CTAB (cetyl-trimethylammonium bromide) method (Sambrook and Russel, 2001). For DNA amplification by the polymerase chain reaction (PCR), the primers ISR fo forward (5' ACCGACGCTTATCGCAGGTCACTA-3') and

Send correspondence to A.C. Vargas. Laboratório de Bacteriologia, Universidade Federal de Santa Maria, Campus UFSM, prédio 44, sala 5137, 97105-900 Santa Maria, RS, Brazil. E-mail: agueda.vargas@gmail.com. 
ISR

reverse

(5'-GTGTCGAAGCA

AAATCAGGGTCGT-3') were used. Fragments with a length of $650 \mathrm{bp}$ corresponding to $M$. bovis and those with a length of $600 \mathrm{bp}$ corresponding to M. bovoculi and M. ovis were observed (Angelos and Ball, 2007). For the differentiation of Moraxella species, the enzyme RsaI was used, which only cleaves the amplified DNA sequence from $M$. bovoculi $(600 \mathrm{bp})$ at a single restriction site, resulting in two fragments of approximately 150 and $450 \mathrm{bp}$. This enzyme does not cleave the DNA fragment of $M$. bovis (650 bp) or M. ovis (600 bp) (Angelos and Ball, 2007). Ten strains of Moraxella spp. were identified as M. bovis, 11 as M. bovoculi derived from cattle, and 11 as $M$. ovis strains isolated from sheep (Table 1).

\section{Broth microdilution method}

The MIC (minimal inhibitory concentration) and MBC (minimal bactericidal concentration) were investigated for ampicillin, gentamicin, neomicin, penicillin, cefoperazone, ceftiofur, cloxacillin, enrofloxacin, florfenicol and oxytetracycline. The MIC was determined in 96-well microplates, in which $100 \mu \mathrm{L}$ of the bacterial inoculum $\left(10^{5} \mathrm{cfu} / \mathrm{mL}\right)$ was added to $100 \mu \mathrm{L}$ of Muller Hinton broth (cation adjusted with $20 \mathrm{~g} / \mathrm{L}$ of calcium and $10 \mathrm{~g} / \mathrm{L}$ of magnesium), in this volume, 12 different concentrations of each antimicrobial agent were diluted for $32 \mathrm{mg} / \mathrm{mL}$ to $0.015 \mathrm{mg} / \mathrm{mL}$. All strains were analyzed in triplicate along with reference strains (M. bovis ATCC 10900, M. bovoculi ATCC BAA1259 and M. ovis ATCC 19575). Staphylococcus aureus ATCC 25923 was used as the standard quality control strain. Finally, the microplates were incubated under aerobic conditions at $35^{\circ} \mathrm{C}$ for $24 \mathrm{~h}$ (CLSI, 2013).

To determine the MBC, $10 \mu \mathrm{L}$ of each antimicrobial dilution corresponding to each strain, equal or higher than the MIC value, was transferred to Muller-Hinton agar plates and followed by incubation at $35^{\circ} \mathrm{C}$ for $24 \mathrm{~h}$.

As no standardized criteria for the interpretation of sensitivity exist for Moraxella spp., breakpoints established for Gram-negative pathogens related with bovine respiratory disease were used. No established breakpoints were available for cloxacillin, cefoperazone and neomycin (CLSI, 2013). The MIC was defined as the lowest concentration of antibiotics that completely inhibited growth, and the $\mathrm{MIC}_{50}$ and $\mathrm{MIC}_{90}$ were defined as the lowest concentrations of antibiotics capable of inhibiting the growth of 50\% and $90 \%$ of the Moraxella spp. isolates, respectively. The $\mathrm{MBC}_{50}$ and $\mathrm{MBC}_{90}$ have been defined as the lowest concentrations of antimicrobial agents at which no bacterial growth is evident in $50 \%$ and $90 \%$ of isolates, respectively (CLSI, 2013).

\section{Statistical analysis}

The MIC and MBC values were evaluated via the Kruskal-Wallis test and subjected to the calculation of posi-

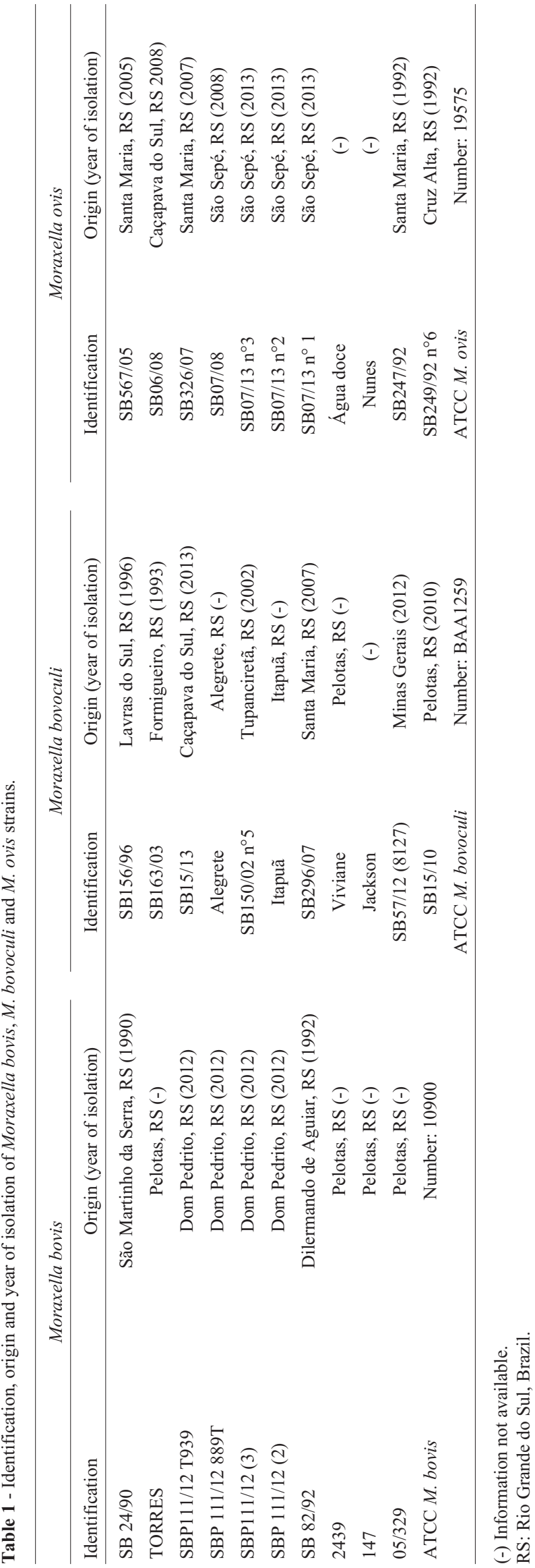


tion measurements. When differences between species of Moraxella were identified, the Bonferroni test was applied to compare the modal MIC values.

\section{Results and Discussion}

The results of the broth microdilution method for the 32 strains of Moraxella spp. are shown in Table 2. Gen- erally, the MIC and $\mathrm{MBC}$ values were similar, indicating that the same concentrations of the antimicrobial agents were able to both inhibit bacterial growth and kill the microorganism (Table 2). Although there are no guidelines to define MICs for Moraxella spp, interpretative criteria derived from other pathogens have been proposed (Angelos et al., 2011). For instance, the critical breakpoints for determining ampicillin, ceftiofur, enrofloxacin, florfenicol, gen-

Table 2 - Minimum inhibitory and bactericidal concentrations $\left(\mathrm{MIC}_{50}, \mathrm{MIC}_{90}, \mathrm{MBC}_{50}\right.$ and $\left.\mathrm{MBC}_{90}\right)$ modal/MIC and resistance of Moraxella bovis, $M$. bovoculi and M. ovis.

\begin{tabular}{|c|c|c|c|c|c|c|}
\hline \multirow[b]{2}{*}{ Antimicrobial agents } & \multicolumn{6}{|c|}{ Moraxella bovis } \\
\hline & $\mathrm{MIC}_{50}$ & $\mathrm{MIC}_{90}$ & $\mathrm{MBC}_{50}$ & $\mathrm{MBC}_{90}$ & Modal-MIC $(\mu \mathrm{g} / \mathrm{mL})$ & $\%$ resistence \\
\hline Ampicillin & 0.125 & 2 & 0.125 & 2 & 0.015 & 0.0 \\
\hline Cefoperazone & 4 & 32 & 4 & 32 & 32 & $\dagger$ \\
\hline Ceftiofur & 0.125 & 1 & 0.125 & 2 & 0.125 & 0.0 \\
\hline Cloxacillin & 2 & $>32$ & 2 & $>32$ & 32 & $\dagger$ \\
\hline Enrofloxacin & 0.03 & 0.06 & 0.06 & 0.06 & 0.0156 & 0.0 \\
\hline Florfenicol & 1 & 1 & 1 & 1 & 1 & 0.0 \\
\hline Gentamicin & 0.125 & 1 & 0.125 & 1 & 0.0156 & 0.0 \\
\hline Neomycin & 0.5 & 1 & 1 & 2 & 1 & $\dagger$ \\
\hline Oxitetracycline & 0.5 & 8 & 0.25 & 2 & 0.25 & 20.0 \\
\hline \multirow[t]{2}{*}{ Penicillin } & 0.25 & 2 & 0.25 & 2 & 0.125 & 40.0 \\
\hline & \multicolumn{6}{|c|}{ Moraxella bovoculi } \\
\hline Antimicrobial agents & $\mathrm{MIC}_{50}$ & $\mathrm{MIC}_{90}$ & $\mathrm{MBC}_{50}$ & $\mathrm{MBC}_{90}$ & Modal-MIC $(\mu \mathrm{g} / \mathrm{mL})$ & $\%$ resistence \\
\hline Ampicillin & $<0.015$ & $<0.015$ & $<0.015$ & $<0.015$ & 0.0156 & 0.0 \\
\hline Cefoperazone & 0.25 & 4 & 1 & 4 & 0.25 & $\dagger$ \\
\hline Ceftiofur & $<0.015$ & 0.03 & $<0.015$ & 0.03 & 0.0156 & 0.0 \\
\hline Cloxacillin & 0.06 & $>32$ & 0.125 & $>32$ & 0.0625 & $\dagger$ \\
\hline Enrofloxacin & 0.03 & 1 & 0.06 & 0.125 & 0.0312 & 0.0 \\
\hline Florfenicol & 0.5 & 1 & 1 & 1 & 0.25 & 0.0 \\
\hline Gentamicin & 0.5 & 1 & 0.5 & 2 & 0.5 & 0.0 \\
\hline Neomycin & 1 & 2 & 2 & 2 & 1 & $\dagger$ \\
\hline Oxitetracycline & 0.5 & 12 & 2 & 2 & 0.25 & 0.0 \\
\hline \multirow[t]{2}{*}{ Penicillin } & $<0.015$ & $<0.015$ & $<0.015$ & $<0.015$ & 0.0156 & 9.0 \\
\hline & \multicolumn{6}{|c|}{ Moraxella ovis } \\
\hline Antimicrobial agents & $\mathrm{MIC}_{50}$ & $\mathrm{MIC}_{90}$ & $\mathrm{MBC}_{50}$ & $\mathrm{MBC}_{90}$ & Modal-MIC $(\mu \mathrm{g} / \mathrm{mL})$ & $\%$ Resistence \\
\hline Ampicillin & $<0.015$ & $<0.03$ & $<0.015$ & $<0.015$ & 0.015 & 0.0 \\
\hline Cefoperazone & 4 & 16 & 8 & 16 & 2 & $\dagger$ \\
\hline Ceftiofur & 0.06 & 0.25 & 0.06 & 0.25 & 0.015 & 0.0 \\
\hline Cloxacillin & 0.5 & 1 & 2 & 4 & 1 & $\dagger$ \\
\hline Enrofloxacin & 0.06 & 0.125 & 0.25 & 0.25 & 0.125 & 0.0 \\
\hline Florfenicol & 1 & 2 & 1 & 2 & 0.5 & 0.0 \\
\hline Gentamicin & 0.5 & 1 & 1 & 1 & 0.5 & 0.0 \\
\hline Neomycin & 1 & 2 & 1 & 2 & 1 & $\dagger$ \\
\hline Oxitetracycline & 0.5 & 4 & 2 & 8 & 0.5 & 9.0 \\
\hline Penicillin & $<0.015$ & 0.5 & $<0.015$ & 0.5 & 0.0156 & 18.0 \\
\hline
\end{tabular}

$\dagger$ Cannot be calculated; no defined breakpoints. 
tamicin, penicillin and oxytetracycline efficacy against respiratory pathogens of cattle (Pasteurella multocida, Mannheimia haemolytica, and Haemophilus somnus) could also represent interpretative data of M. bovis, $M$. bovoculi and M. ovis susceptibility to these antimicrobials. According to these interpretative criteria, most Moraxella spp. strains were considered susceptible to most antimicrobials, however some strains were considered resistant to penicillin and oxytetraciline, such as $M$. bovis strains that showed $40 \%(4 / 10)$ of resistance to penicillin and $20 \%$ $(2 / 10)$ of resistance to oxytetracycline $(>2)$. Other studies found M. bovis strains, isolated in the United States, resistant to gentamicin and oxytetracicline (Shryock et al., 1998). Moreover, M. bovis strains resistant to erythromycin were also reported in South America (Conceição et al., 2004).

According to the interpretative criteria used in this study, 9\% (1/11) of $M$. bovoculi strains could be considered resistant to penicillin. A previous study reported similar results for $M$. bovoculi, indicating higher resistance to penicillin (12.3\%) in comparison with other tested antimicrobials (Angelos et al., 2011). The data reported in the veterinary literature regarding the susceptibility of Moraxella spp. predate the description of $M$. bovoculi, consequently, it is mainly available from studies involving $M$. bovis. (Webber et al., 1982; Shryock et al., 1998; Zielinski et al., 2002; Conceição et al., 2004). In this way, the antimicrobial susceptibility of $M$. bovoculi was only evaluated after the recent description of this species (Angelos et al., 2007), in a study in the United States (Angelos et al., 2011). Thus, this is the second study evaluating the susceptibility profile of $M$. bovoculi.

Some $M$. ovis strains examined in this study could be considered resistant to oxytetracycline $(9 \%-1 / 11)$ and penicillin $(18 \%-2 / 11)$. The susceptibility data for $M$. ovis available in the literature are scarce; the only study addressing the antimicrobial susceptibility of $M$. ovis examined isolates from cattle and reported resistance only for erythromycin (Catry et al., 2007). One reason for this lack of information may be that this pathogen is not the primary agent involved in the etiology of the disease in sheep (Dagnall, 1994). It is important to note that the present report is the first to describe the susceptibility of $M$. ovis derived from sheep, and this etiological agent should also be controlled by antibiotics, especially to avoid exacerbation of lesions primarily caused by $M$. conjunctivae and $C$. pscittaci (Dagnall, 1994).

Oxytetracycline is usually the first choice for antimicrobial treatment of IK (Alexander, 2010). The $\mathrm{MIC}_{50}$ and $\mathrm{MIC}_{90}$ values obtained for oxytetracycline are presented in Table 2. The previously reported MIC values for oxytetracycline for M. bovis (Shryocket al., 1998), M. bovoculi (Angelos et al., 2011), and M. ovis (Catry et al., 2007) are lower than the values reported in this study. Although oxytetracycline is widely used in the treatment of this disease, there are only two reports of resistance of $M$. bovis to this drug in the literature (Shryock et al., 1998; Senturk et al., 2007). In the present study, $20 \%$ of the $M$. bovis strains and $9 \%$ of the $M$. ovis strains could be considered resistant to oxytetracycline. These results may suggest that the indiscriminate use of oxytetracycline over the years can be related with the selection of Moraxella spp. strains resistant to this drug.

We observed significantly high MIC values $(16 \mu \mathrm{L} / \mathrm{mL}$ to $>32 \mu \mathrm{L} / \mathrm{mL})$ for cloxacillin. Susceptibility of M. bovis for cloxacillin was reported (Webber et al., 1982), and strains displaying high MIC values, similar to those found in the present study, were considered resistant. The $\mathrm{MIC}_{90}$ values for florfenicol obtained for $M$. bovis, $M$. bovoculi and $M$. ovis were $1 \mu \mathrm{L} / \mathrm{mL}, 1 \mu \mathrm{L} / \mathrm{mL}$ and $2 \mu \mathrm{L} / \mathrm{mL}$, respectively. A previous study found $3.5 \%$ of resistance for florfenicol among M. bovoculi strains (Angelos et al., 2011). $\mathrm{MIC}_{90}$ values for florfenicol, similar to those found in this study, were reported for M. bovis strains isolated in Argentina (Zielinski et al., 2002) and M. ovis strains isolated in Belgium (Catry et al., 2007). Similar to oxytetracycline, florfenicol has been reported to be an effective treatment option for combating bovine IK (Gocke et al., 2002; Angelos et al., 2011), especially in cases where M. bovis is resistant to tetracycline antibiotic class (Angelos et al., 2000).

The statistical analysis showed that the highest modal values of MIC occurred among the M. bovis strains. In contrast, $M$. bovoculi displayed the lowest modal values of MIC (Table 2). Based on the results obtained using the broth microdilution it can be suggested that there is difference $(p<0.05)$ between the antimicrobial profile of $M$. bovis and those of $M$. bovoculi and M. ovis. Further studies are necessary to determine the reason that higher concentrations of antimicrobials are required to achieve inhibition of $M$. bovis. According to the interpretative criteria used, the in vitro results demonstrate that the three Moraxella species showed the best susceptibility profile for ampicillin, ceftiofur, enrofloxacin, florfenicol and gentamicin.

\section{References}

Alexander D (2010) Infectious bovine keratoconjunctivitis: A review of cases in clinical practice. Vet Clin North Am Small Anim Pract 26:487-503.

Angelos JA, Ball LM (2007) Differentiation of Moraxella bovoculi sp. nov. from other coccoide moraxellae by the use of polymerase chain reaction and restriction endonuclease analysis of amplified DNA. J Vet Diagn Invest 19:532-534.

Angelos JA, Ball LM, Byrne BA (2011) Minimum inhibitory concentrations of selected antimicrobial agents for Moraxella bovoculi associated with infectious bovine keratoconjunctivitis. J Vet Diagn Invest 23:552-555.

Angelos JA, Dueger EL, George LW et al. (2000) Efficacy of florfenicol for treatment of naturally occurring infectious bovine keratoconjunctivitis. J Am Vet Med Assoc 216:6264. 
Angelos JA, Spinks PQ, Ball LM et al. (2007) Moraxella bovoculi sp. nov., isolated from calves with infectious bovine keratoconjunctivitis. Int J Syst Evol Microbiol 57:789-795.

Baptista PJHP (1979) Infectious bovine keratoconjunctivitis: a review. Br Vet J 135:225-242.

Catry BFB, Boyen F, Baele M et al. (2007) Recovery of Moraxella ovis from the bovine respiratory tract and differentiation of Moraxella species by tDNA-intergenic spacer PCR. Vet Microbiol 120:375-380.

Clinical and Laboratory Standards Institute (2013) Antimicrobial disk and dilution susceptibility tests for bacteria isolated from animals. Approved Standard - 4 edition. CLSI document VET01-A4, Wayne, PA.

Conceição FR, Bertoncelli DM, Storch BO et al. (2004) Antibiotic susceptibility of Moraxella bovis recovered from outbreaks of infectious bovine keratoconjunctivitis in Argentina, Brazil and Uruguay between 1974 and 2001. Braz J Microbiol 35:364-366.

Dagnall GJ (1994) The role of Branhamella ovis, Mycoplasma conjunctivae and Chlamydia psittaci in conjunctivitis of sheep. Br Vet J 150:65-71.

Elad D, Yeruham I, Bernstein M (1988) Moraxella ovis in cases of infectious bovine keratoconjunctivitis (IBK) in Israel. J Vet Med B 35:431-434.

Gokce HI, Citil M, Genc O (2002) Comparison of the efficacy of florfenicol and oxytetracycline in the treatment of naturally occurring infectious bovine keratitis. Ir Vet J 55:573-576.
Henson JB, Grumbles LC (1960) Infectious bovine keratoconjunctivitis. I. Etiology. Am J Vet Res 21:761-766.

Libardoni F, Scherer CFC, Farias L et al. (2012) Moraxella bovoculi em casos de ceratoconjuntivite infecciosa bovina no Rio Grande do Sul. Pesq Vet Br 32:743-746.

Macfaddin JF (2000) Biochemical testes for identification of medical bacteria, Lippincott Williams \& Wilkins, Philadelphia, P.A.

Sambrook J, Russel D (2001) Molecular cloning: a laboratory manual, Cold Spring Harbor Laboratory, New York, N.Y.

Senturk S, Cetin C, Temizel M et al. (2007) Evaluation of the clinical efficacy of subconjunctival injection of clindamycin in the treatment of naturally occurring infectious bovine keratoconjunctivitis. Vet Ophthalmol 10:186-189.

Shryock TR, White DW, Werner CS (1998) Antimicrobial susceptibility of Moraxella bovis. Vet Microbiol 61:305-309.

Webber JJ, Fales WH, Selby LA (1982) Antimicrobial susceptibility of Moraxella bovis determined by agar disk diffusion and broth microdilution. AntimicrobAgentsChemother 21, 554-557.

Zielinski GC, Piscitelli HG, Perez-Monti H et al. (2000) Antibiotic Sensitivity of an Argentine Strain Collection of Moraxella bovis. Vet Ther 3:199-204.

Associate Editor: Odir Antonio Dellagostin

All the content of the journal, except where otherwise noted, is licensed under a Creative Commons License CC BY-NC. 